ARGONNE NATIONAL LABORATORY

9700 South Cass Avenue, Argonne, Illinois 60439

ANL-ET/01-13

\title{
MODELING AND SIMULATION OF FRAGMENTATION OF SUDDENLY HEATED LIQUID METAL JETS
}

\author{
by \\ A. Hassanein and I. Konkashbaev \\ Energy Technology Division \\ Computational Physics and Hydrodynamics Section
}

May 2001

\author{
Work sponsored by \\ U.S. DEPARTMENT OF ENERGY \\ OFFICE OF SCIENCE
}




\section{CONTENTS}

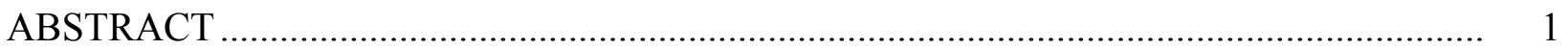

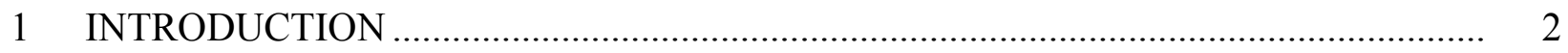

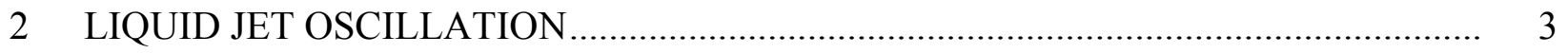

2.1 Radial Oscillations …………………………................................................. 5

2.2 Axial Oscillations ................................................................................................. 7

3 CAVITY APPEARANCE ........................................................................... 9

4 SHOCK WAVE RELAXATION ...................................................................... 12

5 CAVITY DYNAMICS - SPHERICAL CASE ....................................................... 16

6 CAVITY DYNAMICS - CYLINDRICAL CASE .................................................. 19

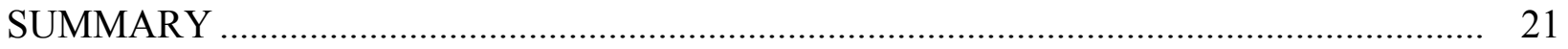

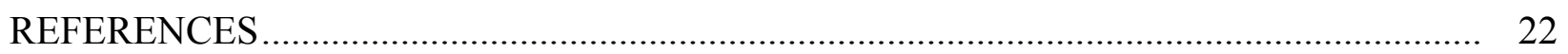

\section{FIGURES}

1 Schematic illustration of interaction between proton beam and liquid metal jet ............... 3

2 Volumetric beam energy deposition in liquid Hg jet........................................................ 4

3 Radial peak temperature distribution after beam deposition ............................................ 6

4 Time dependence of jet radial oscillations ……………………..................................... 6

5 Time dependence of jet surface velocity ……………………………………………..... 7

6 Fluctuations in jet radius with time following beam deposition.......................................... 7

7 Time dependence of radial pressure waves and Hg jet radius ............................................. 7

8 Time dependence of radial density and $\mathrm{Hg}$ jet radius........................................................ 7

9 Time dependence of edge coordinate (length $\mathrm{L}$ ) and velocity of $\mathrm{Hg}$ jet ........................... 8

10 Change in $\mathrm{Hg}$ jet axial length after beam deposition....................................................... 8 
11 Time dependence of distance $\mathrm{z}$ and axial pressure in $\mathrm{Hg}$ jet ................................... 8

12 Time dependence of distance $\mathrm{z}$ and axial density fluctuations in $\mathrm{Hg}$ jet........................ 8

13 Maximum pressure and time of first accumulation waves on axis as function of deposition time when target radius was $0.7 \mathrm{~cm}$ and total energy deposition was $20 \mathrm{j} / \mathrm{g} \ldots . \quad 9$

14 Schematic illustration of relaxation shock wave initiated when cavity appears ............. 13

15 Liquid dynamics after relaxation shock wave ................................................. 15

16 Time dependence of distance 2 and axial cold pressure inside plane jet...................... 15

17 Time dependence of length and axial velocity of plane jet ........................................ 15

18 Dynamics of cavity growth in $\mathrm{Hg}$ target............................................................. 17

19 Variation of cavity radius and velocity as function of time ........................................ 17

20 Time dependence of spherical radius of liquid target and velocity of target surface after cavity initiation ..................................................................... 17

21 Relative target density at outer and inner cavity surfaces after cavity initiation.............. 18

22 Time dependence of radial density after cavity initiation ..................................... 18

23 Time dependence of radial velocity after cavity initiation ..................................... 18

24 Time dependence of radial pressure waves after cavity initiation............................... 18

25 Elongated hole formed by merging spherical cavities............................................. 19

26 Cavity radius and velocity as a function of time after cavity initiation in cylindrical case

27 Oscillations of target surface radius and velocity as a function of time after beam

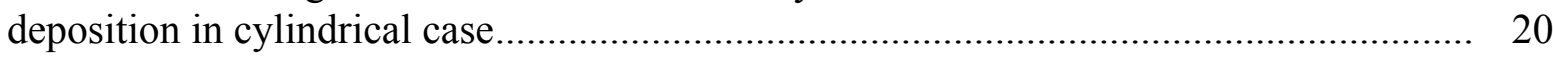

28 Time dependence of cavity and cylinder growth................................................ 20 


\title{
MODELING AND SIMULATION OF FRAGMENTATION OF SUDDENLY HEATED LIQUID METAL JETS
}

by

\author{
A. Hassanein and I. Konkashbaev
}

\begin{abstract}
Thermoelastic response of liquid metal targets exposed to high-volumetric-energy deposition in times shorter than the target hydrodynamic response time (i.e., sound travel time) is of interest to several research areas, including first walls of fusion reactors (especially inertially confined fusion reactors), targets for high-power accelerators such as the Spallation Neutron Source, muon collider targets, etc. Under conditions that exist in these reactors, accelerators, etc., the deposited energy is considered instant in time from the hydrodynamic point of view. Because thermal heat conduction requires a longer than instant response time for energy redistribution, only hydrodynamic phenomena should be taken into account when modeling and simulating the fragmentation of suddenly heated liquid metal jets.

Sudden energy deposition causes an instant rise in temperature that leads to a corresponding rise in the thermal pressure that causes excitation of sound waves, i.e., shock waves and rarefaction waves. During this excitation of sound waves, pressure oscillates with magnitude \pm $\Delta \mathrm{P}$ that corresponds to an initial thermal pressure of tens of katm. Liquids are frequently observed to withstand significant negative pressures (hydrostatic tensile stresses). Yet, a liquid subjected to a negative pressure is metastable. The formation and behavior of cavities (empty voids) under negative pressures was previously studied. Theoretically, the obtained fracture (failure) pressure of mercury is in good agreement with experimental results. Cavitation, or spontaneous formation of cavities, in stressed liquid metal targets is of interest to engineers and physicists who operate high-power targets in fusion reactors, nuclear accelerators, and particle colliders.
\end{abstract}

The problems of liquid target oscillation in the presence of large magnitudes of negative pressure, and the mechanism of fragmentation and its consequences are considered in this analysis. It is shown that a cavity coming into existence will initiate a shock wave that is actually a relaxation shock wave initiated when the stretched medium reverts to normal density from the low-density state. The nature of this relaxation wave is similar to that of the detonation wave. It is also shown that a cavity born at the high-negative-pressure stage expands permanently and does not disappear. This permanent expansion and failure to disappear is a major difference between the cavity dynamics in stretched media and the dynamics observed in the usual cavitation processes that occur when vapor bubbles collapse during a phase of increased pressure, and is the result of "unloading" or "discharging" of the medium by the relaxation shock wave initiated by the appearance of the cavity. Detailed calculations of cavity dynamics are presented for both spherical and cylindrical liquid metal target systems. 


\section{INTRODUCTION}

Thermoelastic response of liquid metal targets exposed to high-volumetric-energy deposition for times shorter than target hydrodynamic response time (i.e., sound travel time) is of interest to investigators engaged in research on, for example, first walls of fusion reactors (especially inertially confined fusion reactors [ICF]), targets for high-power accelerators such as the Spallation Neutron Source, muon collider targets, etc. Under such conditions, the deposited energy is considered instant in time from the hydrodynamic point of view. Because thermal heat conduction requires a longer than instant response time for energy redistribution, only hydrodynamic phenomena should be taken into account, when modeling and simulating the fragmentation of suddenly heated liquid metal jets.

In the muon collider project, for example, the proton beam deposits part of its energy in a liquid metal target, which is thereby heated to a high temperature $\mathrm{T}$ of thousands of degrees, with a corresponding thermal pressure increase $\Delta \mathrm{P}$ of several tens of katm. The energy deposition time $\tau_{\text {energy }}$ of a few nanoseconds is much less than the time of sound travel $\tau_{\text {sound }}$ of tens or hundreds of microseconds: $\tau_{\text {sound }} \approx l / \mathrm{C}_{\mathrm{s}}$, where $l$, the characteristic target size, is $\approx 1-10 \mathrm{~cm}$, and $\mathrm{C}_{\mathrm{s}}$ is the speed of sound of a few $\mathrm{km} / \mathrm{s}$. For such a short deposition time, the deposited energy cannot be spread over the target medium, even by hydrodynamic motion. Therefore, the energy deposition time is considered instantaneous from the target hydrodynamic standpoint. Because thermal conduction requires more time for energy redistribution and mixing, only hydrodynamic phenomena should be taken into account.

Sudden deposition of beam energy in a cylindrical liquid metal target causes an instant rise in thermal pressure, which in turn, causes excitation of sound waves in both the radial direction $r$ and the longitudinal direction $\mathrm{z}$. The corresponding characteristic target response times are $\tau_{\mathrm{R}}=$ $\mathrm{R} / \mathrm{C}_{\mathrm{s}}$ and $\tau_{\mathrm{L}}=\mathrm{L} / \mathrm{C}_{\mathrm{s}}$, where $\mathrm{R}$ and $\mathrm{L}$ are target sizes in the $r$ and $z$ directions. During these excitations, the pressure oscillates with magnitude $\pm \Delta \mathrm{P}$, where $\mathrm{P}_{\max }=\Delta \mathrm{P}$ and $\mathrm{P}_{\min }=-\Delta \mathrm{P}$. Liquids frequently are observed to withstand significant negative pressures (hydrostatic tensile stresses). Yet a liquid subjected to a negative pressure is metastable. Spontaneous formation of cavities in stressed liquid metal targets and the resultant cavitation are of interest to engineers and physicists in the operation of high-power targets in fusion reactors, nuclear accelerators, and particle colliders.

Wave excitation and oscillations due to pressure release and fluid dynamics of a lithium first wall in an ICF reactor were calculated by several authors [1-3]. Liquid target fragmentation was studied only from the standpoint of energetic considerations [4,5] which assumed that part of the deposited energy (corresponding to the deviation of velocities from the mean mass velocity) is converted into surface tension of resultant droplets. However, the problem of which mechanism is mainly responsible for liquid fragmentation and the production of liquid droplets was not properly studied. Nevertheless, various mechanisms that lead to liquid fragmentation do exist and were investigated in the 1940s and 1970s by several authors [6-12]. 
Below, the problems of liquid target oscillation in the presence of large negative pressure, the mechanism of fragmentation, and the consequences are discussed on the basis of previous theoretical work [7], experimental data [8-9], and our HEIGHTS comprehensive numerical simulation package [13-14]. We have developed a new concept of shock wave tensile relaxation that will lead to liquid target fragmentation because of target heating. Most of the numerical calculations are given for a free liquid mercury jet heated by a $20-\mathrm{GeV}$ proton beam, as described in the muon collider project work [15]. The spatial energy deposition in the target was calculated by using the MARS code [16].

\section{LIQUID JET OSCILLATION}

A free liquid metal jet is the assumed target in the production of pions that decay to muons because of interaction with a $20-\mathrm{GeV}$ proton beam, as schematically illustrated in Fig. 1. During beam/target interaction, proton beams deposit a small part of their total energy $(<10 \%)$ in the liquid mercury target. The magnitude of the energy deposited $\mathrm{Q}$ can be rather high, i.e., $\mathrm{Q}=50$ $130 \mathrm{~J} / \mathrm{g}$. Such high-energy deposition leads to instant heating of the target to 1000-2000 K, with a corresponding rise in thermal pressure to 50-100 katm. Pressure relaxation leads to the excitation of both radial and axial oscillations.

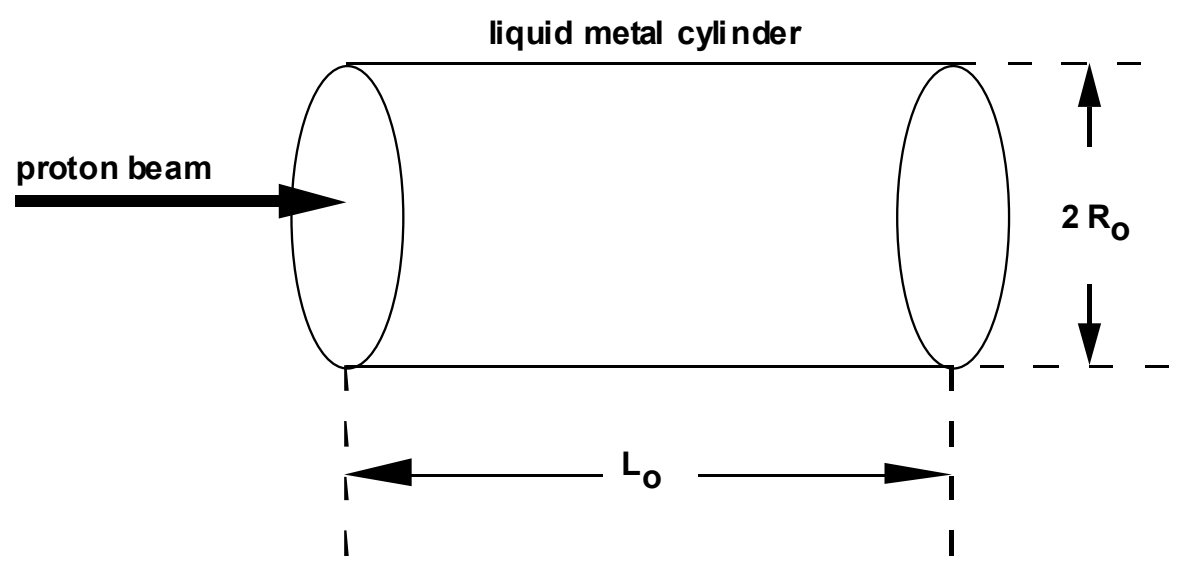

Fig. 1. Schematic illustration of interaction between proton beam and liquid metal jet

The oscillation of the liquid mercury jet in both axial (z) and radial (r) directions were considered separately in this analysis. Because the flight time of the liquid jet along the magnetic field $\tau_{\mathrm{z}}$ of a few milliseconds is much larger than the time of the radial and longitudinal oscillations, the liquid jet is assumed to be stationary in the $\mathrm{z}$ direction during beam/target interaction. The longitudinal motion can, however, be easily taken into account by simply adding a constant-velocity term $U$ along the $\mathrm{z}$ direction, with a corresponding shift in position, by adding $\Delta \mathrm{z}=\mathrm{Ut}$. Most of the results presented in this study are given for the following conditions. The radius of the liquid cylinder $\mathrm{R}_{0}=0.7 \mathrm{~cm}$, the jet length $\mathrm{L}_{0}=29 \mathrm{~cm}$, and two proton beams of the same $20-\mathrm{GeV}$ energy strike the flying jet at $t_{1}=0$ and $t_{2}=150$ ns separately. 
Figure 2 shows the energy deposition distribution in the $\mathrm{z}$ and $\mathrm{r}$ directions as given when we use a fit formula implemented in HEIGHTS to approximate the MARS code calculation.

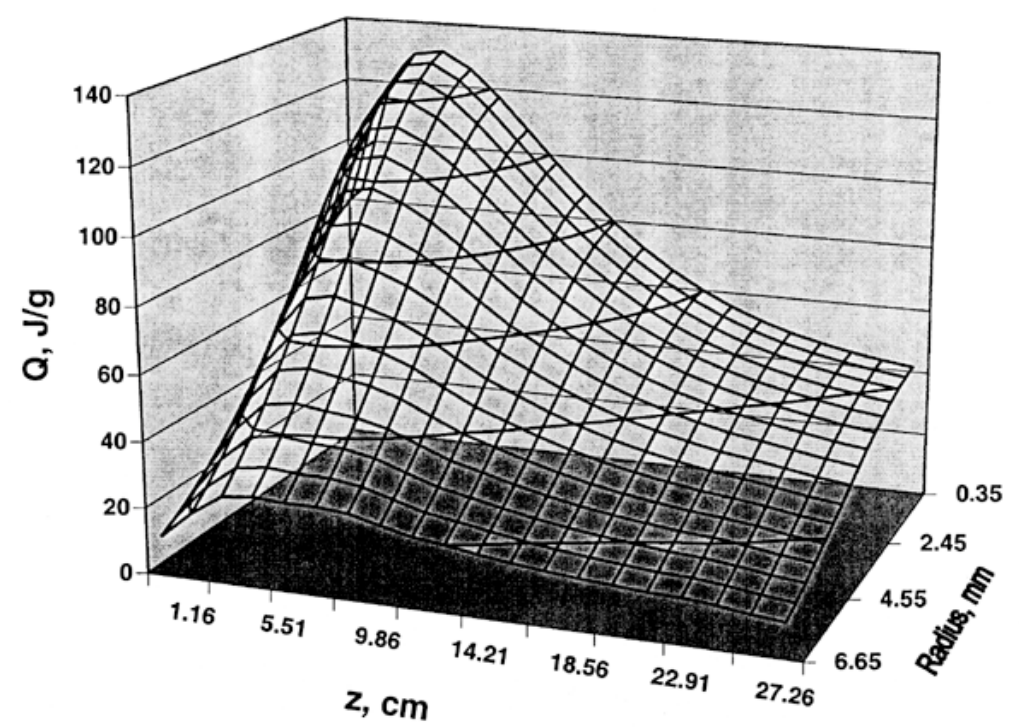

Fig. 2. Volumetric beam energy deposition in liquid Hg jet

For numerical simulations of liquid metal dynamics, we used the comprehensive HEIGHTS MHD package. The following equations of mass, momentum, and energy conservation are solved for the general planar, cylindrical, and spherical target systems:

$$
\begin{aligned}
& \frac{d \rho}{d t}+\mathrm{p} \nabla \cdot \vec{V}=0, \\
& \rho \frac{d \vec{V}}{d t}+\nabla\left(\mathrm{p}+\mathrm{p}_{\mu}\right)=0, \\
& \frac{d E}{d t}+(E+\mathrm{p}) \nabla \cdot \overrightarrow{\mathrm{V}}=0, \\
& \mathrm{p}_{\mu}=\frac{B^{2}}{4 \pi}
\end{aligned}
$$

where $\rho$ is density, $\mathrm{V}$ is velocity, $\mathrm{p}$ is pressure, $\mathrm{E}$ is internal energy of the liquid target, and $\mathrm{B}$ is the applied external magnetic field. The equation of state, which consists of three terms, was used [17], assuming that the internal energy arises from the thermal motion energy of nuclei $E_{i}$, the thermal excitation energy of electrons $\mathrm{E}_{\mathrm{e}}$, and the elastic energy $\mathrm{E}_{\text {cold. }}$. Correspondingly, the pressure also consists of three terms: $\mathrm{P}_{\mathrm{i}}, \mathrm{P}_{\mathrm{e}}$, and $\mathrm{P}_{\text {cold. }}$. 


$$
\begin{aligned}
& E=E_{i}+E_{e}+E_{\text {cold }}(\rho), \\
& E_{i}=3 n k\left(T-T_{o}\right)+E_{o}, E_{e}=\frac{1}{2} \beta_{\mathrm{o}} \sqrt{\rho_{\mathrm{o}} / \rho} T^{2}, \\
& P_{i}=\Gamma(\rho) E_{i}, P_{e}=\frac{1}{2} E_{e}, \\
& \Gamma(v)=-\frac{2}{3}-\frac{v}{2} \frac{d^{2} P_{c o l d}}{d v^{2}} / \frac{d P_{\text {cold }}}{d v}, v=\frac{1}{\rho}, \\
& P_{\text {cold }}=P_{x o}\left[\left(\frac{\rho}{\rho_{\mathrm{o}}}\right)^{n}-1\right], P_{x o}=\frac{1}{n} \rho_{o} C_{\text {so }}^{2},
\end{aligned}
$$

where $T_{0}$ is the initial target temperature; $E_{0}$ is the initial thermal energy; $\beta_{0}$ is the coefficient of electron specific heat; $\Gamma$ is the Gruneisen coefficient determined by the volume dependence of the tensile pressure $\mathrm{P}_{\text {cold }}$, obtained from relevant experiments; power $n$ depends, in general, on density $\rho$; and $C_{\text {so }}$ is the sound speed under initial conditions, $T=T_{0}$, and $\rho=\rho_{o}$.

\subsection{Radial Oscillations}

In the case of the muon collider target that uses a free-flying mercury jet with a velocity of $10-20 \mathrm{~m} / \mathrm{s}$, the flight time from the entrance to the coil is much longer than the magnetic field diffusion time. However, the jet flight time is less than the radial oscillation period caused by the sound wave excitation. Therefore, the electric and magnetic fields were taken into account in cylindrical geometry, assuming that in the initial state, the jet outside the magnetic field that has a magnitude of $\mathrm{B}_{\mathrm{o}}=20 \mathrm{~T}$ and is completely diffused into the liquid cylinder. Equations for the magnetic and electric fields have the form

$$
\begin{aligned}
& \frac{\partial \vec{B}}{d t}=-c \cdot \nabla x \vec{E}, \\
& \vec{E}=\frac{1}{c}[\vec{V} x \vec{B}]+\frac{\vec{j}}{\sigma}, \\
& j=\frac{c}{4 \pi} \nabla x \vec{B},
\end{aligned}
$$

where the magnetic field $\mathrm{B}$ has only a $\mathrm{z}$ component in this case, the electric field $\mathrm{E}$ and the current density $\mathrm{j}$ have only a $\varphi$ component, the velocity $\mathrm{V}$ has only an $\mathrm{r}$ component, and the electric conductivity $\sigma$ of the liquid metal target is assumed constant.

Figure 3 shows the radial distribution of temperature that is a result of beam energy deposition from only one proton beam at the axial distance $\mathrm{z}=5 \mathrm{~cm}$ (where the energy deposition is maximum). The corresponding maximum temperature is $\approx 1200 \mathrm{~K}$ and the thermal pressure is $\approx 50 \mathrm{katm}$. Because of pressure relaxation, the liquid metal cylinder oscillates with 
modes $\mathrm{k}_{\mathrm{r}}=0, \infty$. For a given wide distribution of beam energy deposition, which can be approximated by a Gaussian profile,

$$
Q(r)=\exp \left(-x^{2}\right) x=\frac{r}{\sqrt{2} \lambda}
$$

where the effective size $\lambda$ is $\approx 0.3 \mathrm{~cm}$, in this particular case, most of the deposited energy is concentrated in the main mode with a corresponding frequency $\mathrm{f}_{\mathrm{o}} \approx \mathrm{C}_{\mathrm{s}} / \mathrm{R}_{\mathrm{o}}=10^{5} \mathrm{~Hz}$. For narrower beams, higher excitation modes exist. The time dependence of jet radius and velocity of the cylindrical surface following beam deposition is given in Figs. 4 and 5, respectively. The magnitude of the surface velocity reaches hundreds of meters per second but it is still 10 times slower than the sound velocity $\mathrm{C}_{\mathrm{s}} \approx 2.5 \mathrm{~km} / \mathrm{s}$; therefore, the resultant oscillations are near the linear mode. Figure 6 shows the fluctuation in jet radius with time after beam energy deposition. These oscillations clearly demonstrate the shock wave propagation and reflection at the jet surface. Figures 7 and 8 illustrate the radial and time dependence of pressure $P(r, t)$ and the change of density $\Delta \rho(r, t)$ relative to the normal value $\rho_{o}$, i.e., $\Delta \rho(r, t) / \rho_{o}$, respectively. Hereafter the space coordinates $\boldsymbol{r}$ and $\boldsymbol{z}$ shown in figures that indicate space and time dependence indicate the Lagrangian coordinate, i.e., the coordinate of the given mass at $t=0$. It can be seen in Fig. 8 that the density oscillates around a value determined by the equilibrium between the thermal pressure and the cold pressure (thermal expansion). The temperature oscillates with a magnitude of $\Delta \mathrm{T} \approx 400 \mathrm{~K}$ around an equilibrium value of $900 \mathrm{~K}$. The pressure oscillates with a magnitude of $\approx \Delta \mathrm{P} \approx 40 \mathrm{katm}$; therefore, during a half period of $5 \mu \mathrm{s}$, the liquid negative tension reaches $\approx-40 \mathrm{katm}$. It follows from these calculations during times of interest of tens of microseconds, that the diffusion of magnetic fields can be neglected; therefore, the magnetic field can be assumed frozen in liquid metal and

$$
\frac{B_{z}}{\rho}=\frac{B_{z o}}{\rho_{o}} .
$$

Thus, the space and time dependence of the magnetic field is similar to that of density.

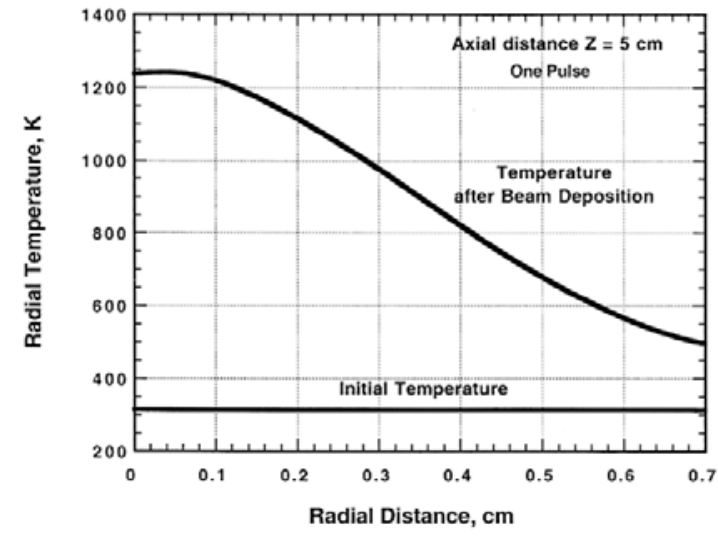

Fig. 3. Radial peak temperature distribution after beam deposition

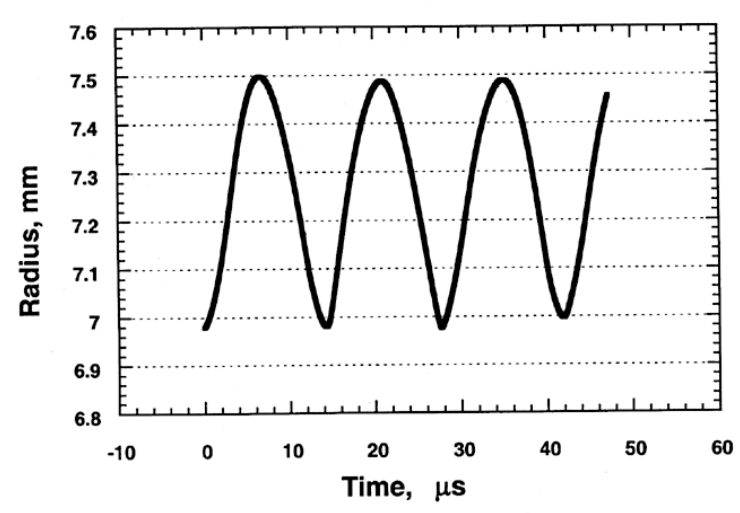

Fig. 4. Time dependence of jet radial oscillations after beam deposition 


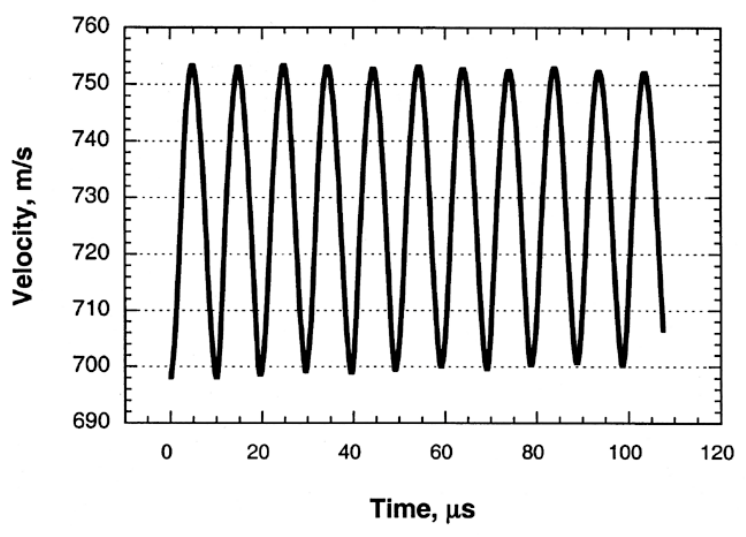

Fig. 5. Time dependence of jet surface velocity

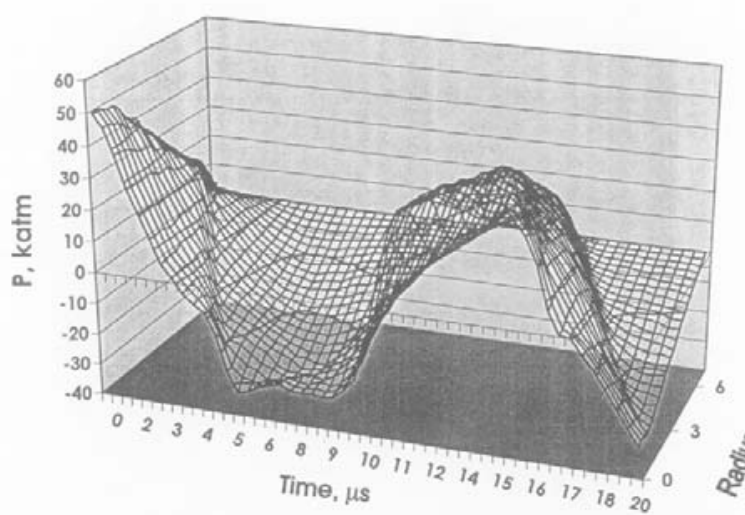

Fig. 7. Time dependence of radial pressure waves and $\mathrm{Hg}$ jet radius

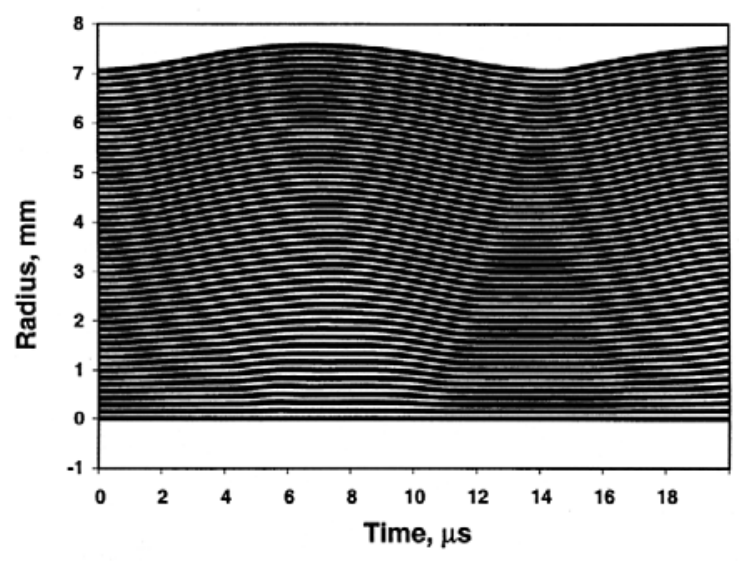

Fig. 6. Fluctuations in jet radius with time following beam deposition

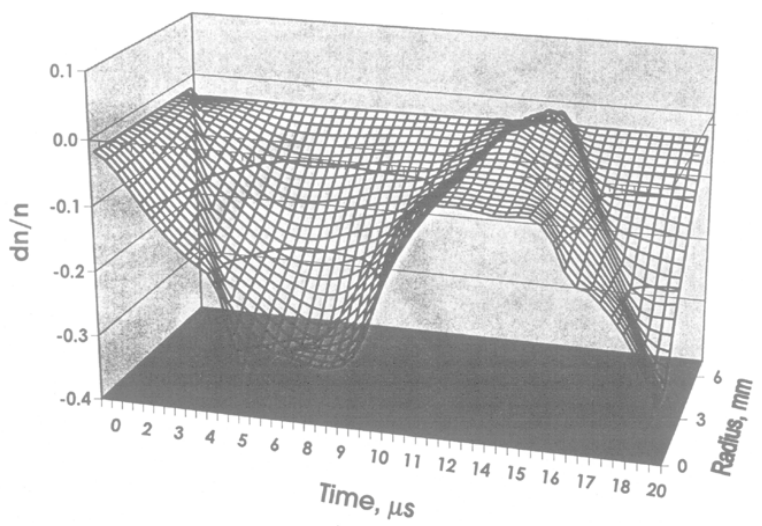

Fig. 8. Time dependence of radial density and $\mathrm{Hg}$ jet radius

\subsection{Axial Oscillations}

The axial magnetic field has no influence on the liquid motion along magnetic field lines; therefore, the axial magnetic field is time independent. The deposited beam energy attains a maximum at $\mathrm{z}=5 \mathrm{~cm}$; therefore, the temperature also attains a maximum at $\mathrm{z}=5 \mathrm{~cm}$. The target liquid cylinder will also oscillate along the $\mathrm{z}$ axis, but with lower frequency than the radial oscillations. Because energy deposition is more or less homogeneous along the axis, most of the energy also concentrates in the main mode with a corresponding frequency $\mathrm{f}_{\mathrm{o}} \approx \mathrm{C}_{\mathrm{s}} / \mathrm{L}_{\mathrm{o}}=10^{4} \mathrm{~Hz}$. The time dependence of the length and velocity of the cylinder surface is shown in Fig. 9. The magnitude of the surface velocity also reaches hundreds of $\mathrm{m} / \mathrm{s}$. Figure 10 shows the time evolution dynamics of the axial length of the jet. Figures 11 and 12 show the space and time dependence of the axial pressure $\mathrm{P}(\mathrm{z}, \mathrm{t})$ and the axial density deviation from the normal value $\rho_{\mathrm{o}}$, i.e., $\Delta \rho(\mathrm{z}, \mathrm{t}) / \rho_{\mathrm{o}}$. The magnitude of the temperature oscillations is $\Delta \mathrm{T} \approx 400 \mathrm{~K}$. The magnitude of the axial pressure oscillations $\Delta \mathrm{P} \approx 30 \mathrm{katm}$, with an equilibrium value of $\approx 20 \mathrm{katm}$; 
therefore, the negative tension reaches only about $-20 \mathrm{katm}$. The period of the axial oscillations is $\approx 500 \mu \mathrm{s}$, increasing correspondingly with the ratio $\mathrm{L}_{0} / \mathrm{R}_{0}$, when compared with the period of the radial oscillations.

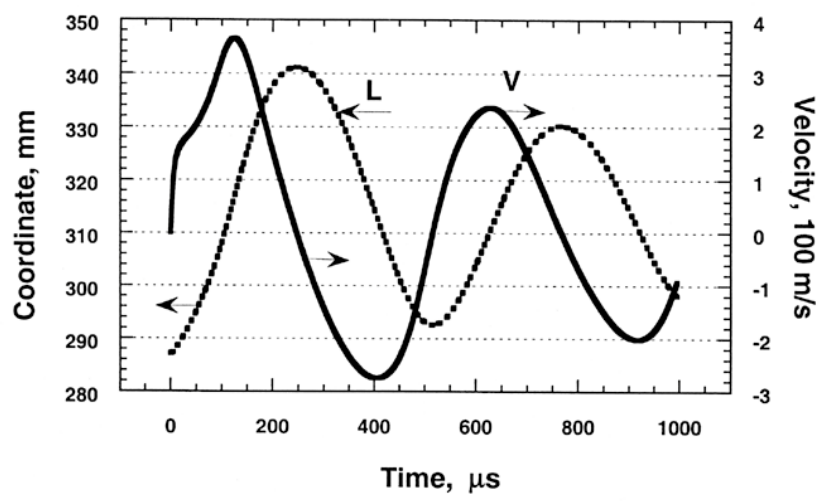

Fig. 9. Time dependence of edge coordinate (length $\mathrm{L}$ ) and velocity of $\mathrm{Hg}$ jet

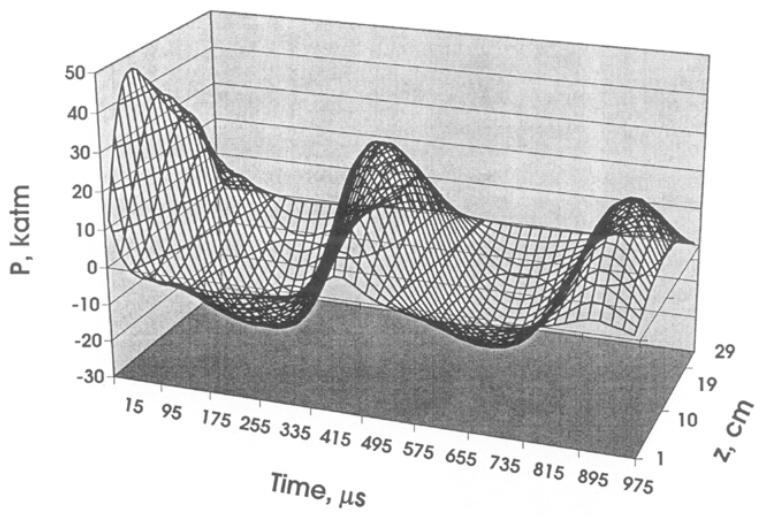

Fig. 11. Time dependence of distance $z$ and axial pressure in $\mathrm{Hg}$ jet

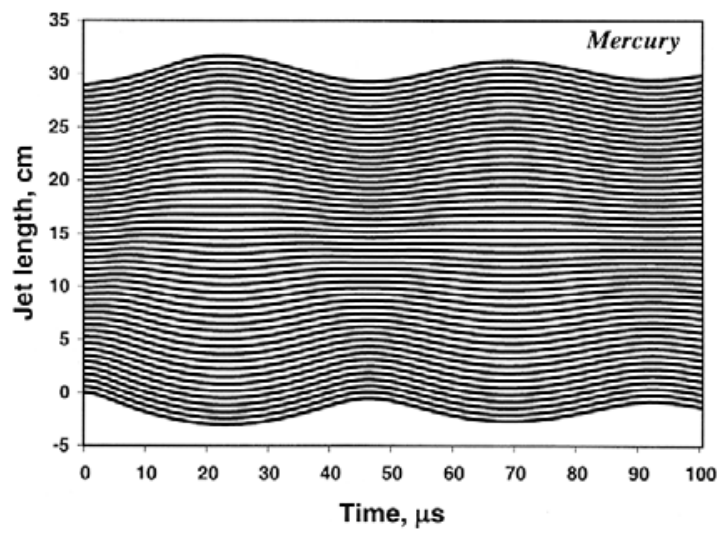

Fig. 10. Change in Hg jet axial length after beam deposition

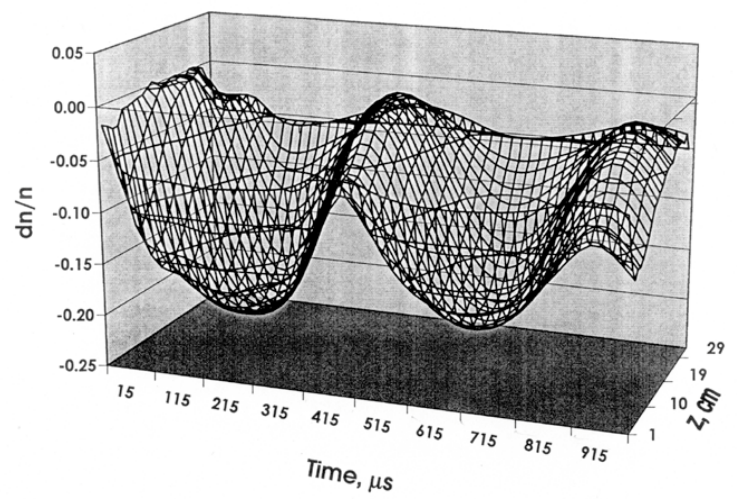

Fig. 12. Time dependence of distance $z$ and axial density fluctuations in $\mathrm{Hg}$ jet

It is important to note that for the equation-of-state given in the form of Eq. 2 with constant power $n$, the maximum negative pressure cannot exceed that of $\mathrm{P}_{\mathrm{xo}}$ because in reality the liquid density at $\mathrm{P}=-\mathrm{P}_{\mathrm{xo}}$ should equal zero, i.e., the liquid must break! Therefore, the calculation for the radial oscillations was made for only one beam interaction inasmuch as the negative pressure approaches $-\mathrm{P}_{\mathrm{x}}$ with the initial thermal pressure of $50 \mathrm{katm}$ because of the cumulative phenomena at the axis in cylindrical geometry. For the axial (planar) case, the negative pressure reaches only $20 \mathrm{katm}<\mathrm{P}_{\mathrm{xo}}$, even for the initial thermal pressure of $100 \mathrm{katm}$ that is the result of the two-beam deposition. 
The definition of fast energy deposition is determined from the relationship between the energy deposition time $\tau_{\text {energy }}$ and the travel time of sound $\tau_{\text {sound, }}$, i.e., $\zeta=\tau_{\text {energy }} / \tau_{\text {sound, }}$, along the target (in $r$ or $z$ directions). At $\zeta<1$, the energy is deposited faster than the time of pressure relaxation along the target by sound waves; therefore, excitation of sound waves (shock and rarefaction waves) takes place. In this case, large negative pressure near the axis can occur because of accumulation of the rarefaction wave. At $\zeta>1$, the energy deposition is slow; therefore, the resultant pressure has time to equilibrate along the target. Such a process is quasistationary when at every time the total pressure $\mathrm{P}(\mathrm{t})$ is near zero, i.e., the thermal pressure is equilibrated by the cold pressure due to material expansion. This process is the well-known phenomenon of material thermal expansion when total $\mathrm{P}(\mathrm{t}) \approx 0$. Figure 13 shows the maximum pressure at the target axis and the time of first accumulation given as a function of the energy deposition time. The calculations in this case were made for a cylindrical graphite target with radius $\mathrm{R}=0.7 \mathrm{~cm}$ and a total energy deposition of $\mathrm{Q}=20 \mathrm{~J} / \mathrm{g}$. The travel time of sound $\tau_{\text {sound }}=$ $\mathrm{R} / \mathrm{V}_{\mathrm{s}} \approx 1.8 \mu \mathrm{s}$ for a sound speed in a carbon target of $\mathrm{V}_{\mathrm{s}}=3.87 \mathrm{~km} / \mathrm{s}$. It can be seen that $\mathrm{P}_{\max }$ sharply decreases from $8 \mathrm{katm}$ to $1 \mathrm{katm}$ with increasing energy deposition time from $\tau_{\text {energy }}=0$ to $1 \mu \mathrm{s}(\zeta \approx 0.5)$ and $\mathrm{P}_{\max }$ approaches zero as $\zeta \rightarrow \infty$. Actually, $\mathrm{P}_{\max }$ can be regarded as near zero at $\zeta>4$. Of course, a result of thermal expansion is that there is no serious problem with target fragmentation. The only problems are target cooling and fatigue lifetime due to thermal cycling effects.

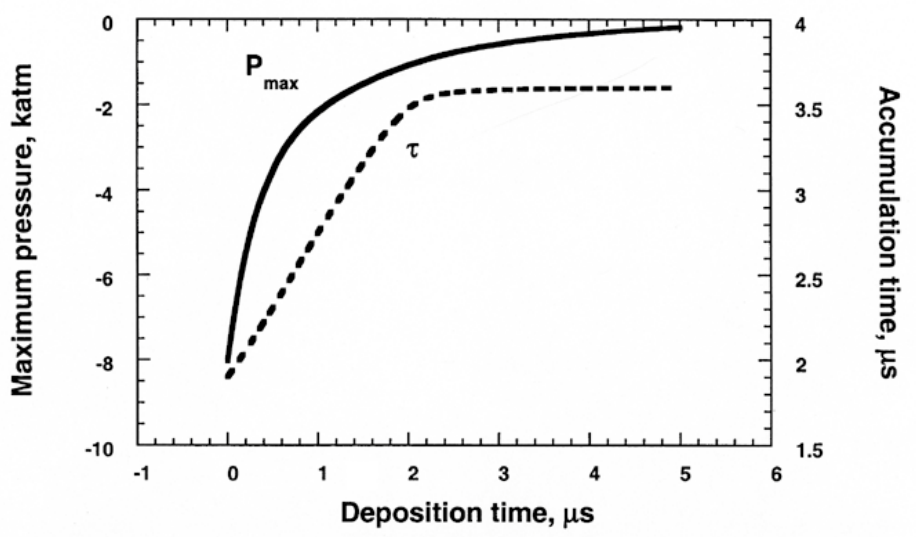

Fig. 13. Maximum pressure and time of first accumulation waves on axis as function of deposition time when target radius was $0.7 \mathrm{~cm}$ and total energy deposition was $20 \mathrm{j} / \mathrm{g}$

\section{CAVITY APPEARANCE}

Let us consider the consequences of these oscillations in relationship to the problem of target break and fragmentation. The above calculations show that the resultant pressure and temperature are rather high and comparable to the critical values for a mercury target, i.e., $\mathrm{T}_{\text {crit }}=$ $1763 \mathrm{~K}$ and $\mathrm{P}_{\text {crit }}=1.535 \mathrm{katm}$. However, the density is still near the normal value $\rho_{\text {norm }}=13.6$ 
$\mathrm{g} / \mathrm{cm}^{3}$ and quite far from the critical density $\rho_{\text {crit }}=5.3 \mathrm{~g} / \mathrm{cm}^{3}$. Therefore, such conditions are far from those necessary for spinodal decomposition and the liquid target remains stable relative to phase change conditions. However, the liquid target under these conditions may be unstable because of existing negative pressure phases in the presence of which the liquid is under severe tension. A main mechanism of possible liquid fragmentation, developed in this work, is the tensile relief wave initiated by the birth of cavities in severely stretched liquids, as will be described below.

There are three main mechanisms of target fragmentation. In the first, after reflection of the shock wave from the free surface boundary, complete destruction and vaporization can take place because of pressure relief and unloading at the surface. However, the magnitude of the required shock wave should be sufficiently high to deliver to target atoms enough energy to exceed the binding energy of a few eV per atom. The magnitude of the required shock for instant fragmentation is quite high and can exceed 1 Matm. The conditions of the shock encountered in the muon collider targets are not that high and this mechanism will not be considered in this study.

A second reason for target fragmentation is the negative pressure exceeding the value $\mathrm{P}_{\mathrm{xo}}$, at which, by definition, the medium should fail. This is a very destructive process, and for solid materials such a limit of tension is measurable. However, solid materials are mostly polycrystalline (i.e., they consist of grains) and measured tension failure is determined by intergrain properties. Only measurements for pure crystalline materials can provide the required data for the ultimate strength. Such measurements exist, however, only for solids and not for liquids.

For liquids subjected to large negative pressures, failure is attributed to preexisting cavities, the third mechanism of fragmentation. Therefore, the mechanism of dynamic arising of cavities and the consequences on materials failure are analyzed in detail in this study. It may be helpful, however, to say a few words about the equation of state at negative pressures. At this time, no fully satisfactory theory exists for calculating the thermodynamic functions of materials with lower-than-normal density when they are subjected to negative pressure. There also are no experimental data. Under these circumstances, experiments on liquid targets with oscillating pressure phases would be very helpful in accessing this failure mechanism. By comparing experimental data with relevant calculations, it is possible to find the actual dependence of the cold and thermal pressures on density and temperature.

The idea behind this liquid fragmentation mechanism is the tensile relief wave that is initiated spontaneously in response to arising cavities. A liquid that is subjected to a strong negative pressure is known to be metastable. Cavitation theory for the vapor bubbles that rise and collapse in an overheated liquid state has been well investigated in the past. But the formation of cavities under strong negative pressure displays features that are different from those suggested by the theory of void nucleation in the superheated state [6-7].

The net work associated with the reversible formation of a spherical cavity of radius $r$ inside a liquid medium subjected to a negative pressure is given by 


$$
W=4 \pi r^{2} \sigma+\frac{4}{3} \pi r^{3}\left(P-P_{v}\right)
$$

where $\mathrm{P}$ is the pressure in the liquid (negative), $\mathrm{P}_{\mathrm{v}}$ is the vapor pressure in the cavity (positive), and $\sigma$ is the surface tension. Because a large negative pressure of several tens of katm is encountered in this analysis, the cavity vapor pressure $\mathrm{P}_{\mathrm{v}}$ can be neglected and therefore the work $\mathrm{W}$ maximum occurs at the critical radius $\mathrm{r}^{*}$, defined as

$$
r^{*}=-\frac{2 \sigma}{P}, W_{\max }=\frac{16 \pi}{3} \frac{\sigma^{3}}{P^{2}}
$$

A cavity coming into existence with a radius less than the critical radius, i.e., $r<r^{*}$, will disappear and cavities with $r>r^{*}$ will grow freely. The equilibrium distribution of cavities with radius $R$ is given by

$$
n(r)=N \exp \left(-\frac{W}{k T}\right),
$$

where $\mathrm{N}$ is the number of particles (atoms, molecules) in the liquid. by

The theory of nucleation [6-7,10-12] predicts that the rate of critical cavity formation is given

$$
\frac{d n}{d t}=\frac{N k T}{h} \exp \left(-\frac{\Delta f+W_{\max }}{k T}\right),
$$

where $\Delta \mathrm{f}$ is the free energy of activation for the motion of an individual particle of liquid away from the cavity surface, i.e., $\Delta \mathrm{f} \approx 5000-7500 \mathrm{cal} / \mathrm{mole}$ [7]. The relative variation of failure pressure $\mathrm{P}_{\tau}$ with $\Delta \mathrm{f}$ is small $(<10 \%)$; therefore, for reasonable estimates, it is convenient to substitute $\Delta \mathrm{f}=0$ without loss of accuracy. From Eq. 9 , one can obtain the value of the fracture pressure as

$$
\begin{aligned}
& P_{\tau}=-\sqrt{\frac{16 \pi}{3 k T} \frac{\sigma^{3}}{\operatorname{Ln} A}} \sqrt{\frac{\operatorname{Ln} A}{\operatorname{Ln} A+\operatorname{Ln} \tau}} \\
& A=\frac{N k T}{h}
\end{aligned}
$$

where $\tau$ is the time within which at least one cavity can be expected to form. For liquid mercury with $\sigma=474 \mathrm{erg} / \mathrm{cm}^{2}$, 


$$
\begin{aligned}
& A=\frac{N k T}{h}=2.55 \cdot 10^{35}, \operatorname{Ln} A=96.24 \\
& P_{\tau}=P_{\tau o} \sqrt{\frac{L n A}{L n A+L n \tau}}, P_{\tau o}=22.3 \mathrm{katm} .
\end{aligned}
$$

Therefore, at negative pressure $\mathrm{P}<-\mathrm{P}_{\tau}$, liquid mercury can be fractured (broken) because of the growth of cavities. It should be noted that $\operatorname{Ln}(\tau)$ is usually much lower that $\operatorname{Ln}(\mathrm{A})$; therefore, $\mathrm{P}_{\tau}$, practically, does not depend on time $\tau$ because of weak logarithmic dependence.

Experiments to study liquid fracture under negative pressure have been carried out with liquid mercury under various conditions [8,9]. Early experiments of Briggs [8] showed that the fracture pressure has a strong dependence on experimental conditions, i.e., cleanliness of the mercury and the surfaces of the glass tube that contain the liquid. The liquid fracture pressure increased from 7 to 425 atm by additional cleaning of the mercury. Further experiments [9], performed under controlled conditions with highly purified mercury, show that the fracture pressure is $\approx 19 \mathrm{katm}$; very similar to the theoretical limit of $22.3 \mathrm{katm}$. The overall agreement between mercury experiments and the theory was very good [9]. The large discrepancy between the earlier data [8] and theory can be explained by mercury that was not sufficiently pure to eliminate dissolved gases and by using a glass tube to eliminate embryonic sites at the glass surface. Practically, the fracture pressure under liquid working conditions is usually five to ten times lower than the theoretical limit due to these dissolved gases and impurities. For example, for water, $\mathrm{P}_{\tau}$ (theory) $=1.32 \mathrm{katm}[6]$, but $\mathrm{P}_{\tau}$ (experimental) is only lower than $350 \mathrm{~atm}$ due to the presence of impurities [9].

Therefore, one can conclude that at negative pressures below the fracture limit, the birth rate of cavities with critical radius is practically independent of the time at which the pressure remains negative. Below, we will consider our new concept of the mechanism of fracture as the consequence of cavities coming into existence during the negative-pressure phase.

\section{SHOCK WAVE RELAXATION}

In this section, we study the dynamics of a liquid target in which a cavity with an initial radius equal to or greater than the critical radius is coming into existence. As mentioned before, the vapor pressure inside the cavity can be neglected and the cavity is regarded as an empty hole. Therefore, inside the empty cavity the pressure is equal to zero, i.e., $\mathrm{P}_{\text {cavity }}=0$, but the liquid outside the cavity is at negative pressure $\mathrm{P}_{\text {liquid }}=-\mathrm{P}$. At the cavity interface, a jump of pressure given by

$$
\Delta P=P_{\text {cavity }}-P_{\text {liquid }}=P
$$

takes place ahead of the cavity boundary; therefore, a shock wave with an amplitude $\mathrm{P}_{\text {shock }}=\Delta \mathrm{P}$ is formed. This shock wave is, in reality, a relaxation wave that occurs when the stretched

medium reverts back from density $\rho<\rho_{o}$ to the normal density $\rho_{o}$. The characteristic of this 
relaxation shock wave is similar to that of a detonation wave because the energy that initiates the shock is taken from within the medium itself, i.e., it releases the energy used in stretching the target. Figure 14 schematically illustrates cavity expansion and the initiated shock wave of relaxation.

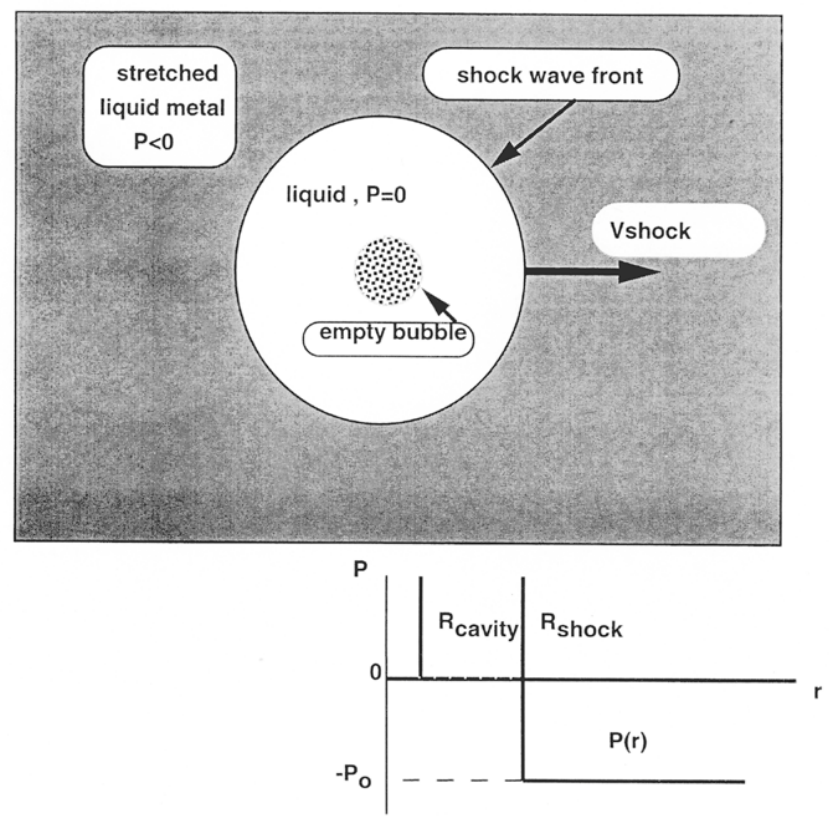

Fig. 14. Schematic illustration of relaxation shock wave initiated when cavity appears

The features of this shock wave of medium relaxation can be clearly demonstrated for the plane geometry case, wherein the jump in pressure is always equal to $\Delta \mathrm{P}$ (from $-\Delta \mathrm{P}$ to zero). The velocity of the medium $U$ between the shock wave and the cavity boundary is constant and can be obtained from the following analytical considerations.

Let us define the states of the medium ahead of (without subscript) and behind (subscript o) the shock front. The mass and momentum conservation laws can be written in the form

$$
\begin{aligned}
& \rho D=\rho_{o}(D-U) \\
& \Delta P=\rho D U
\end{aligned}
$$

where $\mathrm{D}$ is the speed of the shock propagation through the stretched medium. The third equation (energy conservation law) can be written in the form of the Hugoniot equation

$$
\Delta E_{s} \rho=\frac{1}{2} \Delta P\left(\rho_{o}-\rho\right)
$$


where $\Delta \mathrm{E}_{\mathrm{s}}$ is the total energy acquired by the substance because of the shock. The change in the internal energy, in turn, is composed of changes in the elastic and thermal energies. The jump in the elastic energy of the system corresponds to the jump in the density. In the pressure range of interest, tens of katm, the difference between isentropic adiabatic and Hugoniot adiabatic is almost imperceptible because the contribution of the thermal pressure is negligible when compared with that of the cold pressure.

Therefore, all of the elastic energy should be converted into kinetic energy because after the shock front passes the density is equal to the normal density and the corresponding elastic energy is equal to zero:

$$
\frac{1}{2} \rho_{\mathrm{o}} U^{2}(D-U)=\Delta E D
$$

where $\Delta \mathrm{E}$ is the energy of elastic deformation. From Eqs. 13 and 16, one can obtain the velocity $\mathrm{U}$ and the shock wave speed $\mathrm{D}$ :

$$
\begin{aligned}
& U=\sqrt{2 \frac{\Delta E}{\rho}}, D=D_{o} \sqrt{2 \frac{\Delta E}{E_{o}} \frac{\rho_{o}}{\rho}} /\left(1-\frac{\rho}{\rho_{o}}\right) \\
& D_{o}=C_{S o} .
\end{aligned}
$$

Because the deviation in density $\varepsilon$ is much less than unity, i.e., $\varepsilon=\left(\rho_{o}-\rho\right) / \rho_{o}<<1$, one can expand $\mathrm{D}$ in a power series of $\varepsilon$ to obtain

$$
D=D_{o} \sqrt{\frac{1}{1-\varepsilon^{2}}}>D_{o} .
$$

Thus, the shock front moves supersonically as usually observed in such shock waves. The liquid behind the shock wave moves with subsonic velocity, given by

$$
U=C_{s o} \sqrt{\frac{\varepsilon^{2}}{1-\varepsilon}}, U=D \varepsilon
$$

The main difference between this relaxation shock and a conventional shock wave is that the relaxation shock is driven not by external forces, for example, when energy is deposited into the medium from outside, but by the internal source of energy. This shock is similar to a detonation shock wave because the energy source is the stored elastic energy that is released immediately when the shock front arrives.

To illustrate this example, using the HEIGHTS package, we performed a simple calculation for a slab of liquid of size $L=10 \mathrm{~cm}$ stretched $(1+\varepsilon)$ times, where $\varepsilon=0.05$. Figure 15 shows that the size of the slab decreases with constant velocity and the relaxation shock wave moves with supersonic speed. Because $\varepsilon=0.05<<1$, this speed is, however, slightly above the sound velocity. Figure 16 shows how the cold pressure returns back to zero when the density returns to 
the normal value because of the relaxation shock wave. The velocity evolution in the plane geometry case is shown in Fig. 19. The calculated exact value of this velocity, $\mathrm{V}=68.9 \mathrm{~m} / \mathrm{s}$, is in good agreement with the theoretical estimate from Eq.18.

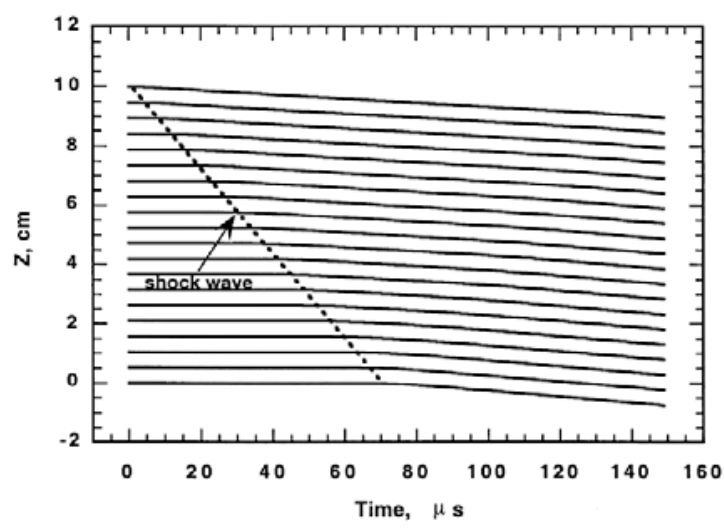

Fig. 15. Liquid dynamics after relaxation shock wave

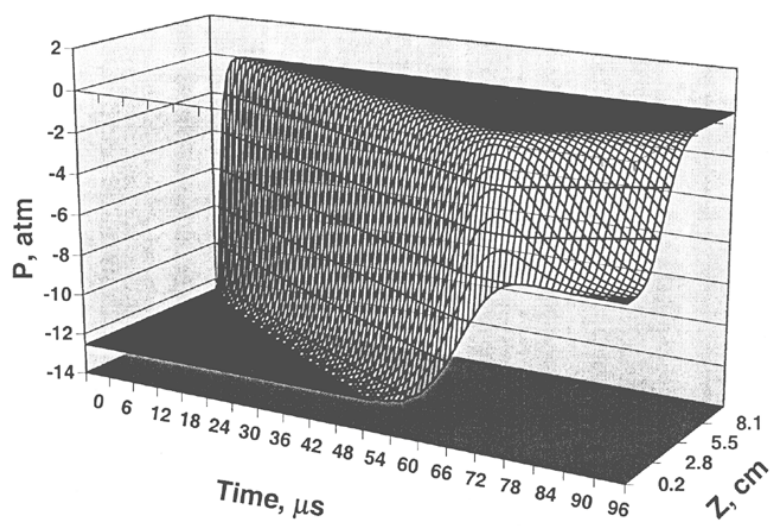

Fig. 16. Time dependence of axial cold pressure inside plane jet

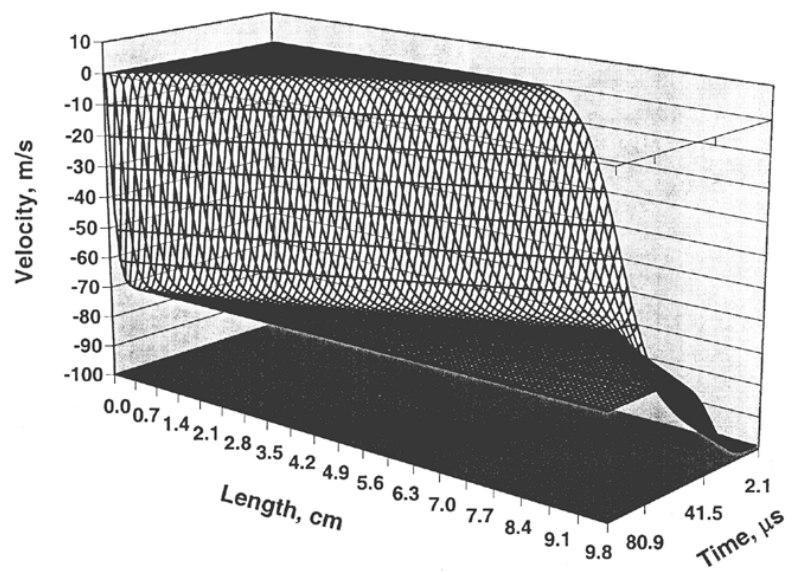

Fig. 17. Time dependence of length and axial velocity of plane jet

The above calculations are similar to those encountered in an ICF reactor, in which a thick (L $=10-20 \mathrm{~cm}$ ) flow of liquid lithium is used to protect the wall from neutron radiation and pellet debris. Because the chamber radius $\mathrm{R}$ (a few meters) is much larger than the flow depth of the liquid lithium, flow simulation dynamics can be considered planar. After the initial shock wave is reflected at the free surface, the rarefaction wave propagates to the back side, leaving behind the stretched liquid. Therefore, during the time of this wave propagation, cavities can grow if the magnitude of the negative pressure exceeds the fracture limit of the liquid target.

For a cylindrical or spherical geometry, it is necessary to take into account that the medium velocity between the shock wave and the cavity boundary is not constant but depends on the radius of the cylinder or sphere. Because the released energy per unit volume is constant, 
additional redistribution of the released energy is required, and in this case analytical consideration becomes very complicated. Therefore, we considered in detail with the HEIGHTS package only those cases of cavity growth dynamics under negative pressures that were more realistic and of greater interest to us.

\section{CAVITY DYNAMICS - SPHERICAL CASE}

A liquid jet will oscillate between cycles of negative and positive pressure after beam deposition. During the negative-pressure period, the pressure can achieve the fracture limit of, for example, $22.3 \mathrm{katm}$ for pure mercury liquid, or less for a working liquid that contains impurities and dissolved gases. Therefore, several cavities with radius equal to or exceeding the critical size can be born and grow. In the usual cavitation theory, vapor bubbles grow until they encounter the high-pressure period during which they collapse, an event that is recognized by the measurement of the acoustic noise. A similar question arises in our problem: does a cavity that is born and continues to grow during the half-period with negative pressure collapse during the second half-period with positive pressure? The answer to this question was studied numerically with the HEIGHTS package in two cases: cavity dynamics in a spherical target case and in a cylindrical-target case. It will be shown below that the situation in liquid targets exposed to large negative pressure is different from the usual cavitation of vapor bubbles.

The simulation and response of cavity dynamics were studied in detail with HEIGHTS in one-dimensional spherical and cylindrical geometry. To answer the question of cavitation, i.e., the possibility of a cavity collapsing during the stage of positive pressure, oscillation of a liquid sphere heated instantly to a high temperature was simulated. Influence of a strong magnetic field (that exists in the muon collider target chamber) was not taken into account in understanding the spherical-cavity expansion.

The initial conditions of this calculation are the following: radius of the spherical target $\mathrm{R}=1$ $\mathrm{cm}$, inner part of the target $\mathrm{r} \leq 0.5 \mathrm{~cm}$ is heated instantly (at $\mathrm{t}=0$ ) with an energy deposition of $\mathrm{Q}$ $=50 \mathrm{~J} / \mathrm{g}$. After target heating, the temperature rises instantly to $\mathrm{T}_{\mathrm{o}}=1100 \mathrm{~K}$, with a corresponding thermal pressure of $\mathrm{P}_{\mathrm{o}}=20 \mathrm{katm}$. At time $\mathrm{t}=0$ two waves propagate from the surface at $\mathrm{r}=0.5 \mathrm{~cm}$ : a shock wave of compression moving radially outward and a rarefaction wave moving to the sphere center. At time $\mathrm{t} \approx 4 \mu \mathrm{s}$, the rarefaction wave reaches the sphere center with a negative pressure at the center $\mathrm{P} \approx-\mathrm{P}_{\mathrm{c}}=40 \mathrm{katm}$. The target temperature at the center is cooled from the initial $1100 \mathrm{~K}$ to $700 \mathrm{~K}$ because of expansion. The magnitude of $\mathrm{P}_{\mathrm{c}}$ is higher than the initial pressure $P_{o}$ because of the cumulative effect at the center of the spherical target.

A cavity with a size $R_{\text {co }}=0.25 \mathrm{~mm}$ is then assumed to be born at the time of maximum negative pressure. Figure 18 shows both the dynamics of the liquid target and the new cavity at the center of the target (at $\mathrm{t} \approx 4 \mu \mathrm{s}$ ) in the $\mathrm{r}$ - $\mathrm{t}$ plane. Figure 19 shows the time dependence of the cavity radius and cavity surface velocity. It can be seen that the velocity of the cavity surface oscillates with a decreasing magnitude from very high, $>1000 \mathrm{~m} / \mathrm{s}$ at $\mathrm{t}=4 \mu \mathrm{s}$, to $<200 \mathrm{~m} / \mathrm{s}$ at $\mathrm{t}=$ $50 \mu \mathrm{s}$. The velocity oscillates around a mean value that decreases with time but never becomes negative. This oscillation can be explained, as mentioned above, by the release of internal 
energy by the propagation of a shock wave of relaxation ahead of the cavity; thus., bringing the medium back to the normal state with normal density. Figure 20 shows the time dependence of the spherical radius of a liquid target and the target surface velocity. It can be seen that the target velocity also oscillates with a decreasing magnitude much less than the velocities at the cavity surface.

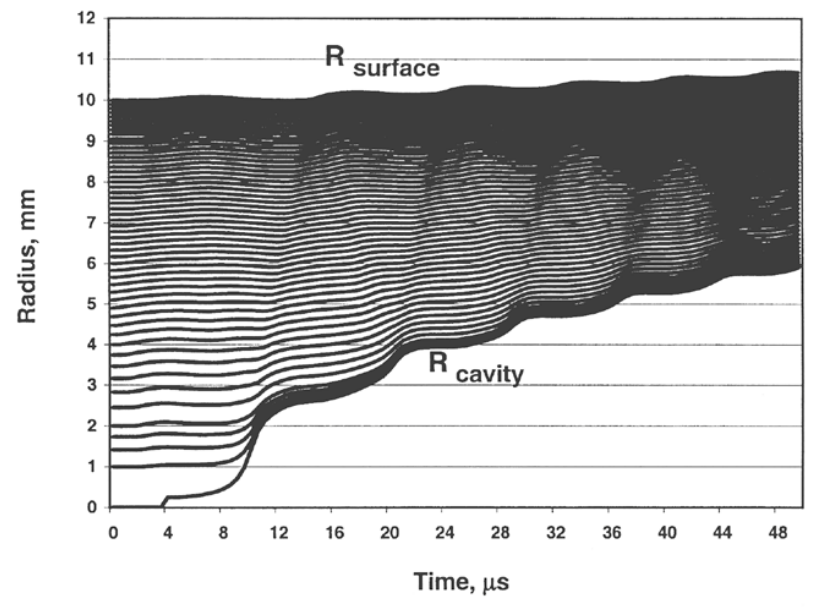

Fig. 18. Dynamics of cavity growth in Hg target

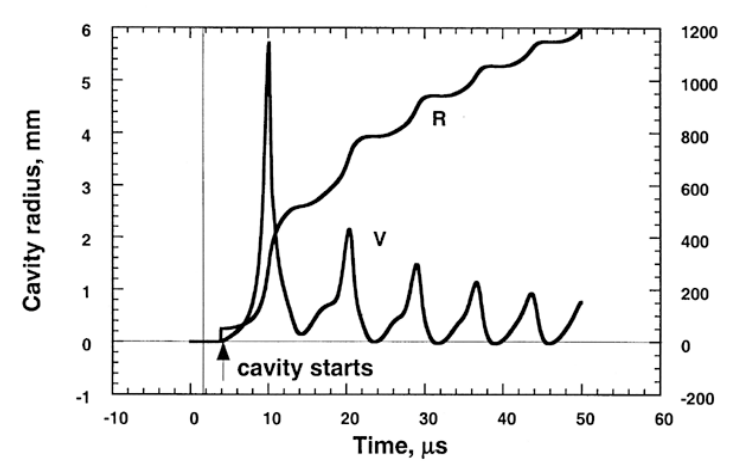

Fig. 19. Variation of cavity radius and velocity as function of time

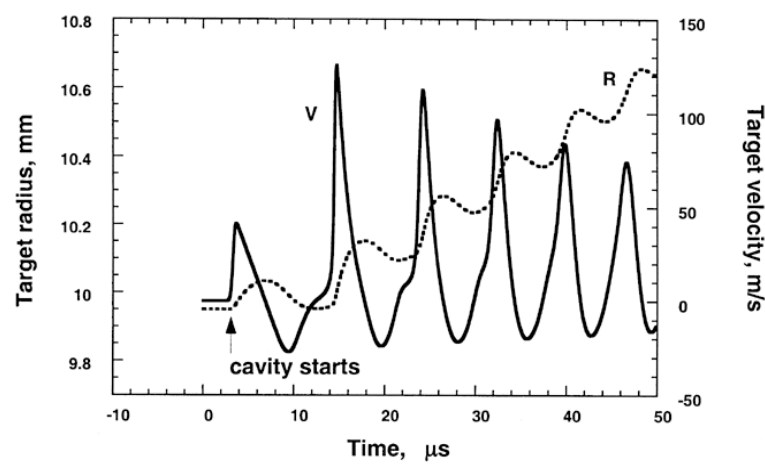

Fig. 20. Time dependence of spherical radius of liquid target and velocity of target surface after cavity initiation

The density at the cavity surface is equal only to $0.8 \rho_{\mathrm{o}}$ because this density is the result of an equilibrium between the thermal pressure and cold pressure at $\mathrm{T}=700 \mathrm{~K}$, as shown in Fig. 21 . Figures 22-24, respectively, show the radial density, velocity, and pressure variation as a function of time. The initial critical size of cavities born at a time during which the absolute 


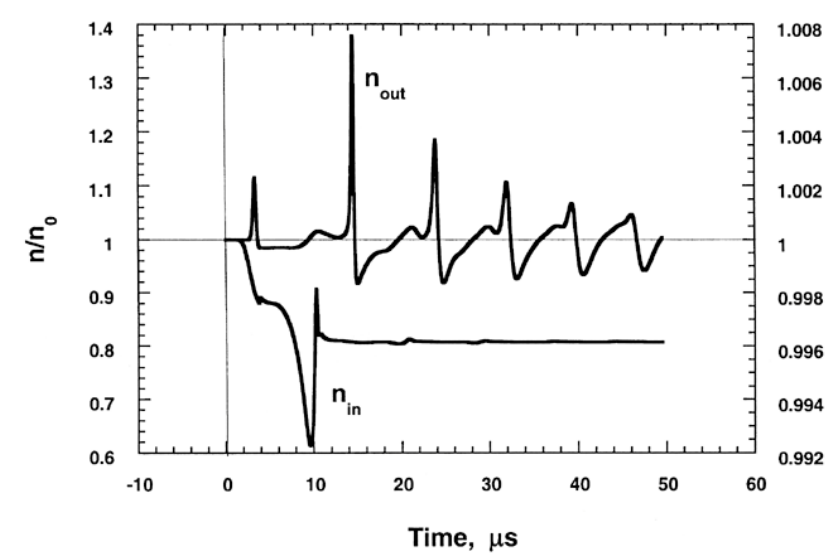

Fig. 21. Relative target density at outer and inner cavity surfaces after cavity initiation

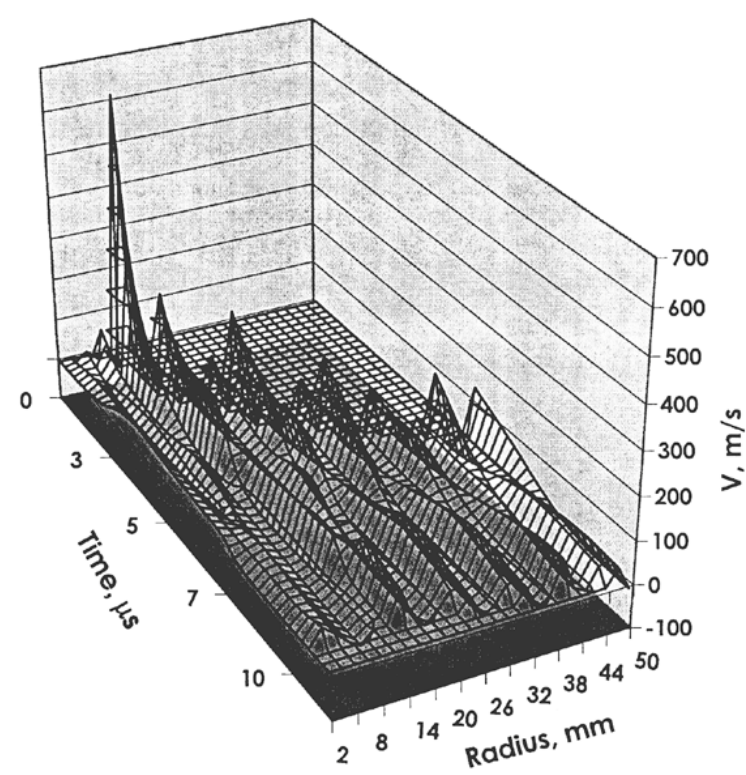

Fig. 23. Time dependence of radial velocity after cavity initiation

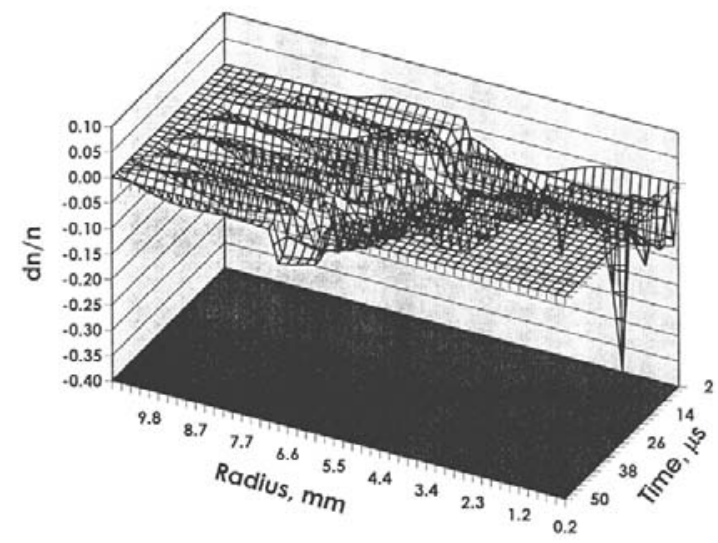

Fig. 22. Time dependence of radial density after cavity initiation

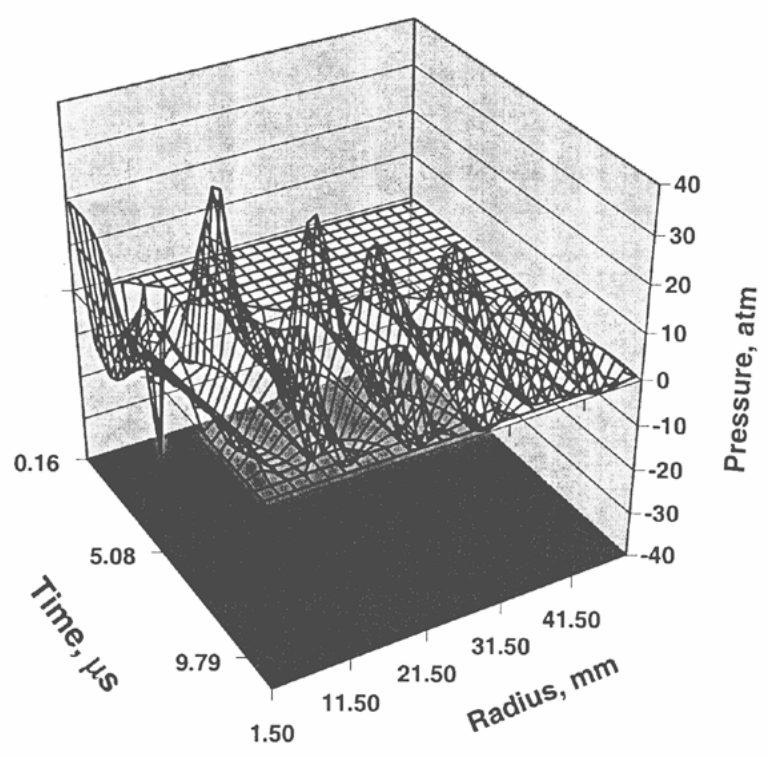

Fig. 24. Time dependence of radial pressure waves after cavity initiation

value of the negative pressure is more than the fracture limit of $22.3 \mathrm{katm}$ (for mercury), will be only $\mathrm{R}_{\text {crit }} \approx 4 \mathrm{~A}^{\mathrm{o}}$ (at $22.3 \mathrm{katm}$ ); therefore, it would be desirable to assume $\mathrm{R}_{\mathrm{co}}=\mathrm{R}_{\text {crit. }}$. However, HEIGHTS simulation shows that the dynamics of cavity growth do not depend on initial size if $R_{\text {co }} \geq R_{\text {crit. }}$ As can be seen in Fig. 19, the velocity of the cavity surface increases rapidly to a very high value that is comparable to the speed of sound. However, with time, the cavity surface velocity decreases and the mean velocity of the shell tends to oscillate around $200 \mathrm{~m} / \mathrm{s}$, which corresponds to the released elastic energy $\Delta \mathrm{E}$. Therefore, to save computer time, the calculations were made with an initial cavity radius of a macroscopic value of $R_{c o}=0.25 \mathrm{~mm}$ because the 
expansion from the critical size to $\mathrm{R}_{\text {co }}$ occurs in a very short time, i.e., $<0.25 \mathrm{~ms}$, and requires a very small computer time-step.

From these calculations, one can conclude that after a cavity is born at the negative pressure phase, it expands freely with a decreasing expansion velocity to a value defined by the elastic energy stored in the system. The spherical target, as a whole, oscillates because of the initial shock, and also expands freely to release its initial energy, which is converted into kinetic energy of the hollow spherical shell. This means that any cavity coming into existence during the negative pressure stage will continue to grow and will not disappear. This failure to disappear or collapse is a major difference between cavity dynamics in a stretched medium and the usual cavitation wherein vapor bubbles collapse during a phase of increased pressure, and is the result of the discharging or unloading of the medium by a relaxation shock wave that is initiated by the appearance of cavities.

\section{CAVITY DYNAMICS - CYLINDRICAL CASE}

The fragmentation of a cylindrical liquid jet is important to the muon collider target. It was shown earlier that the cylindrical jet oscillates in both radial and axial directions after instant beam energy deposition. Because the frequency of the radial oscillations is much higher than that of the axial oscillations, cavity dynamics and fragmentation are mainly governed by the radial oscillations. During a negative pressure phase of tens of microseconds duration, it is highly probable that several cavities can spontaneously come into existence near the jet axis, where the magnitude of the negative pressure is maximum. The dynamics of such cavities will be similar to that of the spherical case discussed above. These cavities also expand freely, initiating a shock wave of relaxation ahead of the cavity surface. During a short time, these cavities will mainly expand in radial directions. However, the expansion velocities of the cavities in both radial and axial directions will be similar. At longer times, these cavities can join to produce an elongated cavity with an axial size that is much larger than the radial one, as schematically illustrated in Fig. 25.

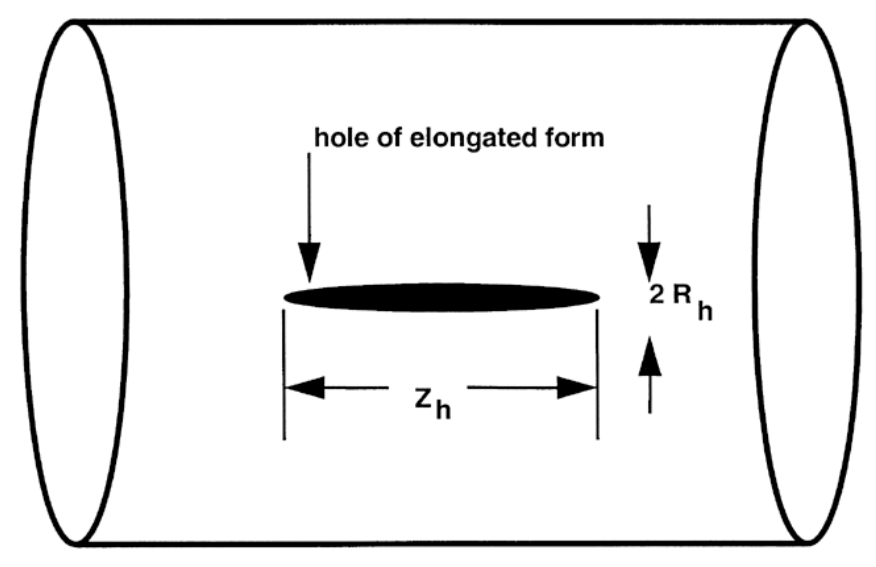

Fig. 25. Elongated hole formed by merging spherical cavities 
Similar calculations were performed with HEIGHTS for conditions relevant to the muon collider mercury target. Figure 26 shows the time evolution of the hole radius $\mathrm{R}_{\text {hole }}$ and surface velocity of the hole $\mathrm{V}_{\text {hole }}$, after the birth of the hole at $\mathrm{t}=4.1 \mu$ s during the negative pressure phase. The cylinder radius $R_{\text {hole }}$ and velocity of the surface $V_{\text {hole }}$ are shown in Fig. 27 as a function of time after beam deposition. The dynamic evolution of the hole and the cylinder is shown in Fig. 28 in the r-t plane. It can be seen that the situation is similar to the spherical case qualitatively and quantitatively. The hollow cylindrical shell expands freely with a rather high mean surface velocity of $\approx 50 \mathrm{~m} / \mathrm{s}$.

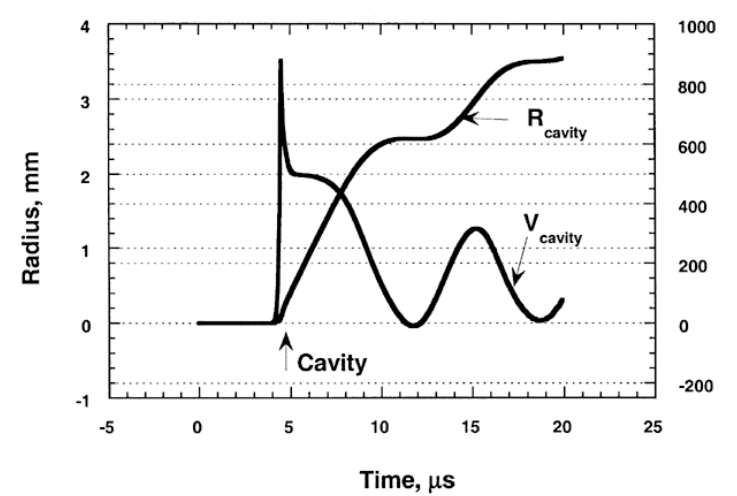

Fig. 26. Cavity radius and velocity as a function of time after cavity initiation in cylindrical case

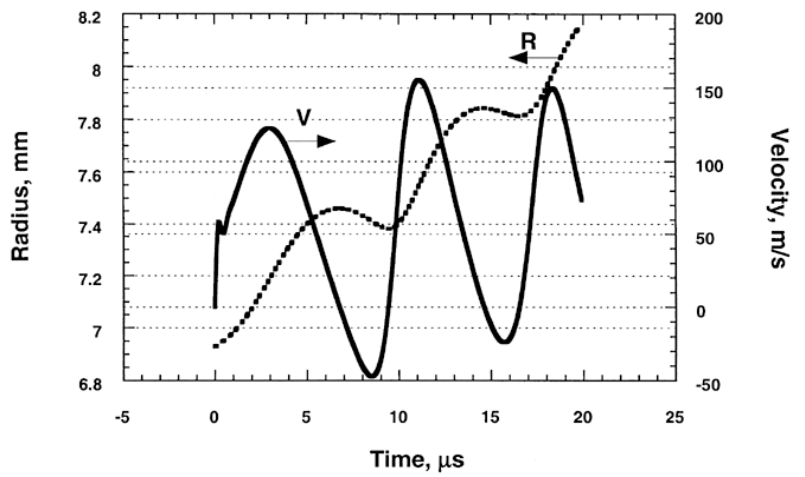

Fig. 27. Oscillations of target surface radius and velocity as a function of time after beam deposition in cylindrical case

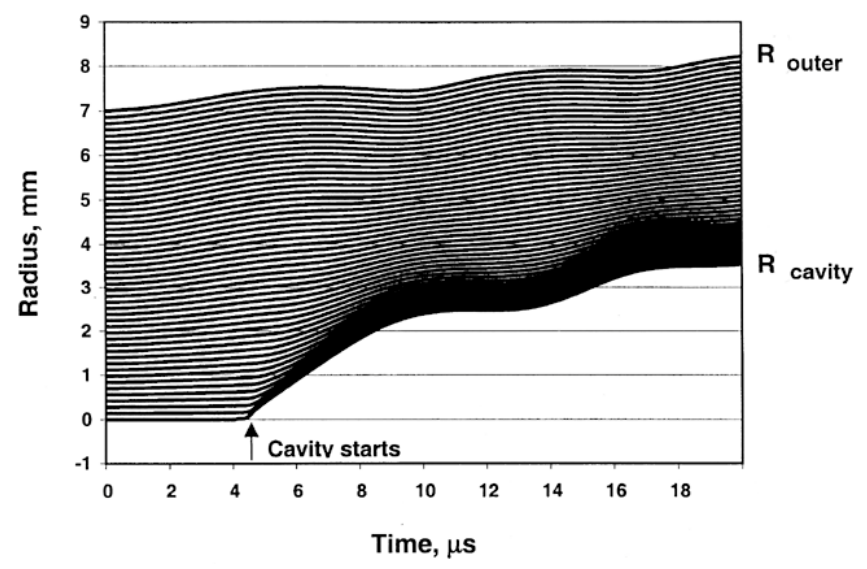

Fig. 28. Time dependence of cavity and cylinder growth

In future studies, the influence of magnetic field on cavity dynamics and liquid fragmentation will be taken into account. However, some speculations about the consequences of such expansion can be made, taking into account the existence of an axial magnetic field. Initially the magnetic field will diffuse from the target medium to the hole or the cavity. However, within tens of microseconds, when the hole expands and its radial size becomes comparable to that of the jet radius, the magnetic field has no time to diffuse inside, and the hole can be regarded as 
free of the magnetic field. Therefore, this situation resembles that of a hollow jet that moves into an external magnetic field. Compression of the magnetic flux lines will result in slowing the liquid metal expansion, i.e., the condition for growth of MHD Rayleigh-Taylor instability. Development of MHD instabilities would lead to the destruction and fragmentation of the liquid jet, forming droplets that splash everywhere with high velocities. Further studies that take into account surface tension and other effects are required.

\section{SUMMARY}

The thermoelastic response of liquid metal targets exposed to intense volumetric energy deposition for short times was studied. The sudden energy deposition causes an instant rise in temperature that leads to a corresponding rise in the thermal pressure that causes excitation of sound waves, i.e., shock waves and rarefaction waves. During these events, pressure oscillates with magnitude $\Delta \mathrm{P}$, corresponding to an initial thermal pressure of tens of katm. Liquids are frequently observed to withstand significant negative pressures (hydrostatic tensile stresses). Yet, a liquid subjected to high negative pressures is metastable and cavities (voids) would arise because of thermodynamic fluctuations.

The dynamics of liquid target oscillation in the presence of large negative pressure, and the mechanism of fragmentation and its consequences are considered. It was shown that a cavity coming into existence initiated a shock wave. This shock wave is actually a relaxation wave when the stretched medium returns from low to normal density. It was also shown that a cavity coming into existence during the negative-pressure stage expands permanently and does not disappear or collapse, even during the positive-pressure phase. The failure to disappear or collapse is a major difference between the cavity dynamics in a stretched medium and usual cavitation, wherein vapor bubbles collapse during a phase of increased pressure, and is the result of the "discharging" or "unloading" of the liquid medium by the relaxation shock wave that is initiated by cavity appearance. Detailed calculations of cavity dynamics were provided for the spherical and cylindrical target cases.

The calculations show that when the absolute value of negative pressure becomes higher than the fracture limit $\mathrm{P}_{\tau}$ (e.g., $\mathrm{P}_{\tau}=22.3 \mathrm{katm}$ for pure mercury), the formation of cavities with an initial size equal to or greater than the critical size $\mathrm{R}_{\text {crit }}$ (4 $\AA$ for mercury at $22.3 \mathrm{katm}$ ) leads to the release of elastic energy with supersonic velocity. Therefore, the entire stretched area with $P$ $<-\mathrm{R}_{\text {crit }}$ reaches a state in which the density corresponds to the common thermal expansion. On the other hand, the negative pressure cannot be $<-\mathrm{P}_{\mathrm{xo}}$ because practically, the liquid as a whole should break. However, no reliable data are available on the thermodynamic properties of liquids at high negative pressure. If the free liquid metal cylinder is instantly heated to high pressures of tens of katm, the negative pressure (especially due to cylindrical cumulative effects) becomes less than the fracture limit, at least near the target axis. Cavities (voids) that come into existence quickly enough initiate relaxation shock waves that release elastic energy in the form of kinetic energy of liquid motion. These cavities soon join together to form an elongated cylindrical cavity. The expansion of this elongated cavity leads to the transformation of the liquid metal cylinder into one or more cylindrical shells that are expanding with a radial velocity determined by the stored potential energy, which is equal to the deposited energy less the 
remaining thermal energy. Hollow cylindrical shells will then expand into the outer strong magnetic field of the muon collider. Compression of the magnetic flux lines will cause the liquid metal to slow down, constituting a condition for the growth of MHD Rayleigh-Taylor instability. Development of MHD instabilities would lead to the destruction and fragmentation of the liquid jet, forming droplets that splash with high velocities. Further studies that take into account surface tension and other effects are required.

\section{References}

1. L. A. Glenn, "On the Motion Following Isochoric Heating of Concentric Liquid Annuli," Lawrence Livermore National Laboratory Report UCRL-84003 (1980).

2. X. M. Chen and V. E. Schrock, "The Pressure Relaxation of Liquid Jets after Isochoric Heating," Fusion Technol., Vol. 19, 1991, pp. 721-726.

3. X. M. Chen, V. E. Schrock, and P. F. Peterson, "The Hydraulic Analysis of Cylindrical Flibe Jets in a HILIFF-II ICF Reactor," Fusion Technol., Vol. 21, 1992, pp. 1451-1535.

4. J. A. Blink, "Fragmentation of Suddenly Heated Liquids," Ph.D. dissertation, and Lawrence Livermore National Laboratory Report UCRL-53604 (1985).

5. L. A. Glenn, "On the Fragmentation of Condensed Material by Isochoric Heating and Release,” Lawrence Livermore National Laboratory Report UCID-19737 (1983).

6. J. C. Fisher, J. H. Hollomon, and D. Turnbull, "Nucleation," J. Appl. Phys., Vol. 19, 1948, pp. 775-784.

7. J. C. Fisher, “The Fracture of Liquids,” J. Appl. Phys., Vol. 19, 1948, pp. 1063-1067.

8. L. J. Briggs, "The Limiting Negative Pressure of Mercury in Pyrex Glass," J. Appl. Phys., Vol. 24, 1952, pp. 488-490.

9. G. A. Carlson, "Dynamic Tensile Strength of Mercury," J. Appl. Phys., Vol. 46, 1975, pp. 4069-4070.

10. M. Volmer, "Kinetik der Phasenbilding," Theodor Steinkopff, Dresden and Leipzig, 1939.

11. J. Frenkel, "Kinetic Theory of Liquids," (Clarendon Press, Oxford, UK 1946).

12. Ja. B. Zeldovich, Acta Physicochem., URSS, Vol. 18, 1943, p. 1.

13. A. Hassanein and I. Konkashbaev, "Fragmentation of Liquid-Metal First Walls in ICF Reactors," Proc. $10^{\text {th }}$ Int. Congress on Plazma Physics (ICPP 2000), Oct. 23-27, 2000, Quebec City, pp. 552-555. 
14. A. Hassanein, "Liquid Metal Targets for High-Power Applications: Pulsed Heating and Shock Hydrodynamics," Laser Particle Beams, Vol. 18, 2000, p. 611.

15. N. V. Mokhov, “The MARS Code System User's Guide, Version 13 (95)," Fermilab FN628 (1995).

16. R. B. Palmer, "Muon Collider Design," Brookhaven National Laboratory Report BNL65242, CAP-200-MUON-98C (Jan. 1998).

17. Ja. B. Zeldovich and Yu. P. Raizer, "Physics of Shock Waves and High-Temperature Hydrodynamic Phenomena" (Academic Press, New York and London, 1967). 
$\underline{\text { Distribution for ANL-ET/01-13 }}$

Internal:

J. Brooks

J. L. Carlson (2)

F. C. Chang

T. H. Chien

H. M. Domanus

H. Drucker
A. Hassanein (20)
I. Konkashbaev (5)
C. A. Malefyt
R. Mattas
J. Norem
R. B. Poeppel

L. Price

W. Shack

R. C. Schmitt

D. Smith

R. A. Valentin

TIS Files

External:

DOE-SC (5)

ANL Libraries:

ANL-E

ANL-W

Main Library:

Fermi National Accelerator Laboratory

Brookhaven National Laboratory

Lawrence Berkeley National Laboratory

Sandia National Laboratories

Los Alamos National Laboratory

Lawrence Livermore National Laboratory

Energy Technology Division Review Committee:

H. K. Birnbaum, University of Illinois at Urbana-Champaign

I.-W. Chen, University of Pennsylvania

F. P. Ford, Rexford, NY

S. L. Rhode, University of Nebraska - Lincoln

H. S. Rosenbaum, Fremont, CA

S. L. Sass, Cornell University

R. Zoughi, University of Missouri-Rolla 